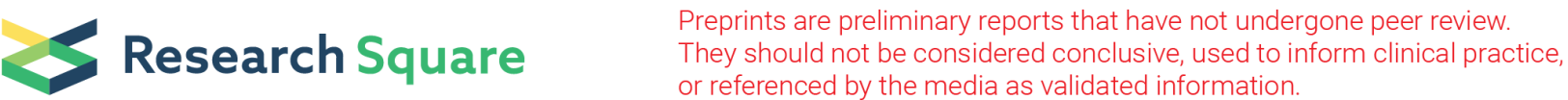

\section{Increased Phosphatase Regenerating Liver -1 Trigger Vascular Remodeling in Injured Ovary Via Platelet-derived Growth Factor Signaling Pathway}

Hyeri Park

CHA University

Jin Seok

CHA University

Jun Hyeong You

CHA University

Jae Yeon Kim

CHA University

Ja-Yun Lim

Korea University

Gi Jin Kim ( $\nabla$ gjkim@cha.ac.kr)

CHA University https://orcid.org/0000-0002-2320-7157

\section{Research}

Keywords: Placenta-derived mesenchymal stem cells, Phosphatase Regenerating Liver-1, Ovary, Folliculogenesis, Vascular remodeling, Platelet-derived growth factor

Posted Date: September 8th, 2021

DOI: https://doi.org/10.21203/rs.3.rs-871700/v1

License: (c) (1) This work is licensed under a Creative Commons Attribution 4.0 International License. Read Full License

Version of Record: A version of this preprint was published at Stem Cell Research \&amp; Therapy on March 7th, 2022. See the published version at https://doi.org/10.1186/s13287-022-02772-9. 


\section{Abstract}

Background: Vascular abnormalities in the ovary cause infertility accompanied by ovarian insufficiency due to a microenvironment of barren ovarian tissues. placenta-derived mesenchymal stem cells (PDMSCs, Naïve) treatment in ovarian dysfunction shows angiogenic effect, however, the therapeutic mechanism between ovarian function and vascular remodeling still unclear. Therefore, we examined whether by phosphatase regenerating liver -1 (PRL-1), which is correlated with angiogenesis in reproductive systems, overexpressed PD-MSCs could maximize the angiogenic effects in an ovarian tissues injured of rat model with partial ovariectomy and their therapeutic mechanism by enhanced vascular function via PDGF signaling.

Methods: PD-MSCs ${ }^{\text {PRL-1 }}$ (PRL-1) were generated by nonviral AMAXA gene delivery system and analyzed the vascular remodeling and follicular development in ovary. One week after Sprague-Dawley (SD) rats ovariectomy, Naïve and PRL-1 was transplanted. The animals were sacrificed at 1, 3 and 5 weeks after transplantation and vascular remodeling and follicular development were analyzed. Also, human umbilical vein endothelial cells (HUVECs) and ovarian explantation culture were performed to prove the specific effects and mechanism of PRL-1.

Results: Vascular structures in ovarian tissues (e.g., number of vessels, thickness and lumen area) showed changes in the Naïve and PRL-1-overexpressed PD-MSC (PRL-1) transplantation (Tx) groups compared to the nontransplantation (NTx) group. Especially, PRL-1 induce to increase the expression of platelet-derived growth factor (PDGF), which plays a role in vascular remodeling as well as follicular development, compared to the NTx. Also, the expression of genes related to pericyte and vascular permeability in arteries was significantly enhanced in the PRL-1 compared to the NTx $(p<0.05)$. PRL-1 enhanced the vascular formation and permeability of human umbilical vein endothelial cells (HUVECs) via activated the PDGF signaling pathway.

Conclusions: Our results show that PRL-1 restored ovarian function by enhanced vascular function via PDGF signaling pathway. These findings offer new insight into the effects of functionally enhanced stem cell therapy for reproductive systems and should provide new avenues to develop more efficient therapies in degenerative medicine.

\section{Introduction}

Vascular development plays a crucial role in follicle maturation and oxygen, nutrient and hormone substrate delivery $[1,2]$. During maturation, the vascular sheath develops along with the maturation of follicles from primordial to secondary follicles and consists of two concentric networks of vessels in the theca interna and externa layers. Arteries and venules branch into the single-layer capillary plexus of the theca interna, but they are not traversed by capillaries into the basement membrane or the granulosa layer. Moreover, the granulosa layer and other cells forming follicles in the ovary receive hormones and nutrients by diffusion from the peripheral theca layer [3]. In particular, vascular endothelial growth factor 
(VEGF) and platelet-derived growth factor (PDGF) are major factors involved in follicular development as well as angiogenic factors. Both VEGF and PDGF play a role in stimulating growth to induce the transition of primordial to secondary follicles [4, 5]. During the menstrual cycle, vascular function degenerates, similar to other organs, and as women age, the major function is lost. Moreover, early vascular dysfunction is considered a factor in women with premature ovarian failure (POF) and ovarian dysfunction. Deficiency of the vasculature in the ovary plays a role in regression and causes ovarian dysfunction by decreasing the diffusion of nutrients and hormones into the follicles. Infertility with side effects (e.g., osteoporosis) occurs due to imbalanced hormone levels through limitation of follicle growth for maturation and acceleration of follicular atresia (i.e., apoptotic follicles) [6]. For the treatment of ovarian dysfunction by aging, hormone replacement therapy (HRT) is mainly used, but this treatment shows temporal effects on ovarian reserve as well as increased risk of various conditions such as breast cancer, cardiovascular disease and osteoporosis [7]. Additionally, whether HRT restores ovarian function through vascular remodeling has not been reported.

To overcome the limitation of HRT due to increased disease risk, many scientists have studied whether the paracrine effects of stem cells, including those mediated by several cytokines [e.g., PDGF, VEGF and fibroblast growth factors (FGFs)] secreted by stem cells, could recover ovarian function in animal models of POF $[8,9]$. Additionally, therapy using stem cells was reported to restore ovarian function, including improvements in follicular development, regulation of reproductive hormones and attenuation of apoptosis [10]. In previous reports, Cho and their colleagues demonstrated that placenta-derived mesenchymal stem cells (PD-MSCs) secreted various growth factors including VEGF and PDGF, and secreted VEGF from PD-MSCs regulates regeneration of vascular remodeling and stimulates follicular development in rats with ovarian dysfunction [11].

Protein tyrosine phosphatases (PTPs) are dual specificity modulators that regulate cell differentiation and proliferation due to their oncogenic effects. In particular, phosphatase of regenerating liver-1 (PRL-1), called PTP4A1, was reported to be involved in liver regeneration [12]. Recently, Schmidt et al. reported that the role of PRL-1 in ovarian function and reported its responses to follicle-stimulating hormone (FSH), which is critical for steroid production and oocyte maturation in the ovary, in primary Sertoli cell cultures [13]. Moreover, PRL deletion limited angiogenesis (e.g., developmental angiogenesis and sprouting angiogenesis) by inhibiting endothelial cell migration and VEGF signaling $[14,15]$. However, the effect of PRL-1 on vascular remodeling in rats with ovarian dysfunction remains unclear.

Therefore, the objective of this study was to demonstrate the therapeutic mechanism by which PRL-1enhanced PD-MSCs affected vascular remodeling as well as ovarian function and whether the paracrine effects of PDGF secretion could improve ovarian function in ovariectomized (OVX) rats.

\section{Materials \& Methods}

\section{Cell culture and gene transfection}


Human placental samples for research purposes were approved by the Institutional Review Board of CHA Gangnam Medical Center, Seoul, Republic of Korea (IACUC-190048). Briefly, PD-MSCs were isolated from the chorionic plate of the placenta as described in previous reports [16]. PD-MSCs and PD-MSCs ${ }^{\text {PRL-1 }}$ were cultured in alpha-minimum essential medium (a-MEM; HyClone, Utah, USA) containing 10\% fetal bovine serum (FBS; Gibco-BRL, Oklahoma, USA), 1\% penicillin/streptomycin (P/S; Gibco-BRL), 25 ng/ml FGF-4 (Peprotech, New Jersey, USA), and $1 \mu \mathrm{g} / \mathrm{ml}$ heparin (Sigma-Aldrich, Missouri, USA) at $37^{\circ} \mathrm{C}$ in a humidified atmosphere of $5 \% \mathrm{CO}_{2}$. For overexpression of human PRL-1 in Naïve PD-MSCs, a human PRL-1 plasmid containing the CMV6-AC vector (Origene, Inc., Rockville, MD, USA) was transfected into Naïve PD-MSCs using the 4D AMAXA Nucleofector ${ }^{\mathrm{TM}}$ system (Lonza, Basel, Switzerland) according to previous reports [17]. After harvest, PD-MSCs were labeled with a PKH67 Fluorescent Cell Linker Kit (Sigma-Aldrich). PD-

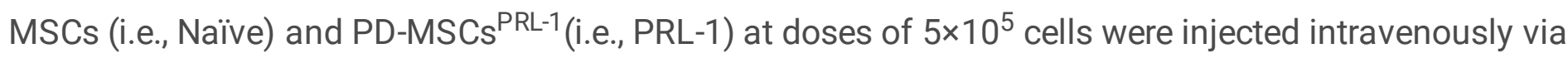
the tail vein in the transplanted animal group.

\section{Construction of the OVX rat model and transplantation of stem cells}

Female 7-week-old Sprague-Dawley rats (Orient Bio, Inc., Seongnam-si, Republic of Korea) were maintained in an air-conditioned animal facility. The rats were housed in groups of two rats per plastic cage with corn-cob bedding and were provided ad libitum access to standard commercial food and tap water. The temperature was $21^{\circ} \mathrm{C}$, and a $12 \mathrm{~h} / 12 \mathrm{~h}$ light-dark cycle was employed. The experimental procedures for the animal modeling and experiments were approved by the Institutional Animal Care and Use Committee of CHA University, Seongnam-si, Republic of Korea (IACUC-190007). The ovariectomy was performed by surgically removing one of the ovaries under general anesthesia with avertin (2,2,2tribromoethanol, Sigma-Aldrich). Each rat was anesthetized through abdominal injection and avertin before the operation. Each group, including the normal, NTx and Tx groups, consisted of 5 rats. One week after OVX modeling, Naïve and PRL-1 ( $5 \times 10^{5}$ cells, 10-13 passages) labeled with a PKH67-linked kit (Sigma-Aldrich) were injected intravenously through the tail vein. All rats were sacrificed after 1, 3, and 5 weeks to harvest their ovarian tissues and blood samples.

\section{RNA isolation and quantitative real-time polymerase chain reaction}

Total RNA isolated from rat ovarian tissues using TRIzol reagent (Ambion, Thermo Fisher Scientific) according to the manufacturing's protocol. Total RNA concentration was measured using a Nanodrop spectrophotometer (Thermo Fisher Scientific, Waltham, USA). Total RNA was reverse transcribed into cDNA using Superscript III reverse transcriptase (Invitrogen). The PCR conditions for the synthesis of cDNA were as follows: $5 \mathrm{~min}$ at $65^{\circ} \mathrm{C}, 1 \mathrm{~min}$ at $4^{\circ} \mathrm{C}, 60 \mathrm{~min}$ at $50^{\circ} \mathrm{C}$, and $15 \mathrm{~min}$ at $72^{\circ} \mathrm{C}$. The cDNA was used for qRT-PCR analysis. It was performed with SYBR Ex Taq (Roche, Basel, Switzerland). The cDNA 
was subsequently amplified by PCR under the following conditions: 5 seconds at $95^{\circ} \mathrm{C}$ and 40 cycles of $95^{\circ} \mathrm{C}$ for 5 seconds and $60^{\circ} \mathrm{C}$ for 30 seconds. The sequences of the qRT-PCR primers are listed in Table S1. rGAPDH was used as an internal control for normalization, and each sample was analyzed in triplicate.

\section{Ovarian explant ex vivo culture}

For ovarian tissue cultivation, Matrigel (Corning, New York, USA) was added to a 24-well culture plate for $3 \mathrm{~h}$. Then, each ovary of 7-week-old female rats was cut and rinsed with saline and DPBS containing $1 \%$ penicillin. For analysis of PRL-1 gene function in ovarian tissues, PD-MSCs treated with siRNA-PRL-1 at $50 \mathrm{nM}\left(1 \times 10^{5}\right.$ cells per ovary; Invitrogen) were directly injected into whole ovarian tissues after ovarian cultivation. After $48 \mathrm{~h}$, the supernatant and ovarian tissues were collected for analysis. Next, to analyze the paracrine effect of PRL-1, an indirect cocultivation system was established using an insert system (Falcon). Naïve or PRL-1 cells were cocultured with the di-sectioned ovaries on an $8 \mu \mathrm{m}$ pore size insert (SPL) and seeded at a density of $1 \times 10^{4}$ cells per insert with medium. For inhibition of PDGF, imatinib mesylate ( $1 \mu \mathrm{M}$; Sigma-Aldrich) was used to inhibit the expression of PDGF, and PDGF recombinant (10 $\mathrm{ng} / \mathrm{ml}$; PeproTech) was used to induce the expression of PDGF on the insert with medium. After $24 \mathrm{~h}$ and $48 \mathrm{~h}$, the supernatant and di-sectioned ovaries were collected, and the samples were analyzed.

\section{Nuclear fraction}

Rat ovarian tissues from each group were measured at $20 \mathrm{mg}$ in a $1.5 \mathrm{ml}$ tube and diluted in $100 \mu \mathrm{l}$ of CER I solution (NE-PERTM Nuclear and Cytoplasmic Extraction Reagents, Thermo Fisher Scientific) with phosphatase inhibitor. Tissues were vortexed and incubated on ice at $10 \mathrm{~min}$. Five microliters of CERII solution (NE-PERTM Nuclear and Cytoplasmic Extraction Reagents, Thermo Fisher Scientific) was diluted in tubes. After vortexing, the samples were placed on ice for $1 \mathrm{~min}$ and centrifuged $(\sim 16000 \times \mathrm{g})$ for $5 \mathrm{~min}$. After centrifugation, the supernatant (cytoplasmic) was transferred to clean prechilled tubes. Pellets were suspended in $50 \mu \mathrm{l}$ of NER solution (NE-PERTM Nuclear and Cytoplasmic Extraction Reagents, Thermo Fisher Scientific). The samples were on ice and vortexed for $15 \mathrm{sec}$ every $10 \mathrm{~min}$ for a total of $40 \mathrm{~min}$. Then, the samples were centrifuged at maximum speed $(\sim 16000 \mathrm{~g})$ in a microcentrifuge for $10 \mathrm{~min}$. The supernatant (nuclear extract) fraction was transferred to clean prechilled tubes.

\section{Protein isolation and western blotting}

Rat ovarian tissues from each group were homogenized and lysed on ice with RIPA buffer (SigmaAldrich) containing protease inhibitor cocktail (Roche) and phosphatase inhibitor cocktail (genDEPOT, Texas, USA). Equal concentrations of protein extracts were separated using $8 \%$ sodium dodecyl sulfatepolyacrylamide gel electrophoresis (SDS-PAGE). The separated proteins were transferred onto polyvinylidene difluoride (PVDF) membranes (Bio-Rad Laboratories, California, USA) using a Transfer 
Turbo system (Bio-Rad Laboratories). Membranes were blocked in blocking buffer (5\% BSA) at room temperature for $1 \mathrm{~h}$. Next, the membrane was incubated with primary antibody $(1: 1000)$ in $2 \% \mathrm{BSA}$ at $4^{\circ} \mathrm{C}$ overnight. The following antibodies were mixed with $2 \%$ BSA and incubated at $4{ }^{\circ} \mathrm{C}$ : rabbit anti-PDGF receptor $\beta$ (28E1; 3169S, Cell Signaling Technology, Danvers, MA, USA) diluted 1:1000, mouse antiPDGFR-a (C-9; sc-398206, Santa Cruz Biotechnology, Dallas, Texas, USA) diluted 1:100, mouse anti-HIF-1a (28b; sc-13515, Santa Cruz Biotechnology) diluted 1:5000, rabbit anti-CD105 (endoglin; bs-4609R; Bioss Antibodies, Woburn, MA, USA) diluted 1:1000, rabbit-anti VEGF receptor 2 (D5B1; 9698S, Cell Signaling Technology) diluted 1:1000, rabbit anti-total mTOR (7C10; 2983S, Cell Signaling Technology) diluted 1:1000, rabbit anti-mTOR (phospho S2448; ab109268, Abcam; Cambridge; MA; USA) diluted 1:1000, rabbit anti-LC3B (2775S, Cell Signaling Technology) diluted 1:1000, mouse anti-Erg-1/2/3 (D-3; sc271048, Santa Cruz Biotechnology) diluted 1:1000, mouse anti-Nobox (D-3; sc-390016, Santa Cruz Biotechnology) diluted 1:1000, rabbit anti-BMP15 (MBS2516631, Mybiosource, San Diego, Ca, USA) diluted 1:1000, and rabbit anti-EGF receptor (2232S, Cell Signaling Technology) diluted 1:1000. After incubation, the membranes were washed with 1X Tris-buffered saline-Tween 20 (TBS-T) and then incubated with secondary antibody (1:10000) according to the manufacturer's instructions at room temperature for $1 \mathrm{~h}$. After the membranes were washed, they were treated with a Clarity Western ECL kit (Bio-Rad Laboratories) at room temperature for $5 \mathrm{~min}$. The protein bands were detected by a ChemiDoc XRS+ imaging system (Bio-Rad Laboratories). The bands were analyzed by the ImageJ program (Wayne Rasband, Bethesda, Maryland). The fold change value of intensity is a comparative value of gene expression.

\section{ELISA}

All blood samples were collected from the aortas of rats in the normal, NTx and Tx groups. Individual serum samples were separated from whole blood by using a blood collection tube. (Vacutainer; BD Biosciences, San Jose, CA, USA). All blood serum was stored at $-80^{\circ} \mathrm{C}$, and estrogen (Biovision, Milpitas, CA, USA), AMH (Elabscience Biotechnology, MA, USA), FSH (Abnova, Taipei, Taiwan), and active caspase3 (Mybiosource, San Diego, CA, USA) activity in serum was analyzed by ELISA kits following the manufacturer's instructions. In brief, an equal volume of sample was added to the specific antibodycoated plates. Next, specific horseradish peroxidase (HRP)-conjugates were added to each well and incubated at $37^{\circ} \mathrm{C}$. After the substrates had been added and incubated in the dark for substrate development, the antibody activity was analyzed by using a microplate reader (BioTek, Winooski, VT, USA).

\section{H\&E staining for follicle counting}

Ovarian tissues were fixed with 10\% neutral buffered formalin (BBC, Washington, USA), embedded in paraffin, and serially sectioned into $4 \mu \mathrm{m}$ ovaries. Sectioned ovarian tissues were deparaffinized in a $60^{\circ} \mathrm{C}$ dry oven and by xylene and ethanol. Deparaffinized tissues were washed under tap water. The slides were 
dipped in Harris hematoxylin (Leica Biosystems, Wetzlar, Germany) for 7 min, dipped in $0.1 \% \mathrm{HCl}$ for 2 seconds and counterstained with alcoholic eosin Y solution (Sigma-Aldrich). The stained slides were scanned for whole ovaries by 3D HISTECH (The Digital Pathology Company, Budapest, Hungary). The follicles were counted every $100 \mu \mathrm{m}$ in serially sectioned slides and were defined as the total follicles, including primordial, primary, secondary, and preovulatory follicles, and the antral follicles according to previous reports [18]

\section{Immunohistochemistry staining}

Sectioned ovarian tissues were deparaffinized in a $60^{\circ} \mathrm{C}$ dry oven and by xylene and ethanol. Deparaffinized tissues were subjected to antigen retrieval by EDTA (eLbio, Seongnam-si, Republic of Korea) reaction and slowly cooled with water. The ovarian tissues were washed with distilled water (D. W) and treated with peroxide blocking solution containing $3 \% \mathrm{H}_{2} \mathrm{O}_{2}$ in methanol for $10 \mathrm{~min}$. Next, the ovarian tissues were washed with D.W and treated with primary antibodies with diluent buffer (Dako) at $4{ }^{\circ} \mathrm{C}$ overnight. The rabbit anti-PDGF receptor $\beta$ antibody (28E1; 3169S, Cell Signaling Technology) was diluted 1:250. After removal of the unbound primary antibody, the tissues in slides were incubated with Dako Real EnVision HRP Rabbit/Mouse secondary antibody (Dako, California, USA) at room temperature for 1 h. The slides were incubated with DAB and counterstained with hematoxylin (Dako). After reaction, slides were rinsed by tap water. Slides were dehydrated by ethanol and xylene. Tissues were analyzed by the 3D HISTECT program (The Digital Pathology Company).

\section{Immunofluorescence staining}

Frozen ovarian section blocks were sectioned at $6 \mu \mathrm{m}$ thickness and fixed with methanol for $10 \mathrm{~min}$. After air drying, ovarian tissues were washed with $1 \mathrm{X}$ phosphate-buffered saline (PBS) at room temperature 3 times for 5 min each. Then, $1 \times$ PBS at the tissue edge was removed and placed in a humidified chamber. Tissues were treated with blocking solution (Dako) at room temperature for $1 \mathrm{~h}$ and treated with primary antibodies against each target gene at $4^{\circ} \mathrm{C}$ overnight. The following antibodies were mixed with antibody diluent buffer (Dako) and used: goat anti-PECAM-1 (CD31; sc-1506, Santa Cruz Biotechnology) diluted 1:250, rabbit anti-LC3B (2775S, Cell Signaling Technology) diluted 1:200, and rabbit anti-phospho-Src (Tyr527, 2105S, Cell Signaling Technology) diluted 1:200. Next, all tissues were incubated at room temperature for $1 \mathrm{~h}$. The tissues were washed with $1 \mathrm{X}$ PBS at room temperature 3 times for $5 \mathrm{~min}$ and then treated with secondary antibody $(1: 250)$ at room temperature for $1 \mathrm{~h}$. The cells were washed with $1 \mathrm{X}$ PBS at room temperature for 5 min 3 times. Then, the tissues were mounted with mounting medium with DAPI (Vectashield, Burlingame, CA, USA). The prepared slides were observed by fluorescence microscopy (Zeiss LSM 780, Oberkochen, Germany) at 400x and 630x magnification. All parts of each slide were observed, and representative images were captured.

\section{HUVEC permeability dextran assay for vascular function}


HUVECs were cultured with ECM medium (Science Cell, California, USA) at $37^{\circ} \mathrm{C}$ in a $5 \% \mathrm{CO} 2$ incubator. Matrigel was coated on an insert with a $0.4 \mu \mathrm{m}$ pore size (Falcon, New York, USA). After 3 h, HUVECs at a dose of $3 \times 10^{5}$ cells were seeded on an insert with a $0.4 \mu \mathrm{m}$ pore size with medium. After $24 \mathrm{~h}$, cells were treated with $100 \mu \mathrm{g} / \mathrm{ml} 5$-FU. After treatment for $48 \mathrm{~h}$, the medium was changed, and Naïve and PRL-1overexpressing cells were seeded in 24-well plates (Falcon). After coculture for $24 \mathrm{~h}$, inserts were transferred to new wells with medium, and dextran (Sigma-Aldrich) was added to the insert. After 20 min, medium on a 24-well plate was loaded in a 96-well assay black plate (Costar, Washington, USA), and the intensity was read with a Tecan assay.

\section{Statistical analysis}

All experiments were conducted in duplicate or triplicate. The results are presented as the mean \pm standard error. Student's t-test was used to analyze the groupwise comparisons. GraphPad Prism 5.0 (GraphPad Software, Inc., CA, USA) was used to conduct statistical analysis using one-way ANOVA followed by Tukey's multiple comparisons test. $P$-values less than 0.05 were considered significant.

\section{Results}

\section{PRL-1-enhanced PD-MSC transplantation increased the expression of PDGF-BB and PDGFR $\beta$ and activation of the PDGF signaling pathway}

The development of follicles in ovarian tissues parallels the development of blood vessels, and abnormal development of blood vessels reduces ovarian function [19]. To examine the structure of blood vessels after PRL-1-enhanced PD-MSC transplantation, we analyzed ovarian sections from different groups by hematoxylin and eosin (H\&E) staining. In the nontransplantation (NTx) group, we observed that blood vessels were elongated compared to those in the normal group. The restoration of blood vessel structure was observed in the transplantation (Tx) group compared to the NTx group; the blood vessel structure changed from an uneven wall to a regular circular wall (Fig. 1a). To analyze the digitization of the blood vessel structure, we measured the number of vessels, the thickness of the arteries, and the lumen area of the vessels. The number of vessels was increased between the NTx and Tx groups including Naïve and PRL-1 groups compared to the normal group. However, the number of vessels was not significantly different between the groups (Fig. 1b). Interestingly, the thickness of the artery and lumen area were substantially increased in the NTx groups compared to the normal group and significantly reduced in the Tx groups compared to the NTx groups (Fig. 1c, d; ${ }^{\star} p<0.05$ ). All Tx groups had vascular structures in the ovaries similar to those of the normal group. These data suggest that transplanted PRL-1-enhanced PDMSCs changed the structure of blood vessels in the ovaries of the OVX rats.

A previous report suggested that transplanted PD-MSCs improved ovarian function via vascular remodeling through the VEGF pathway [11]. Hence, we analyzed the differences between the Naïve and 
PRL-1 groups to determine whether changes in vascular remodeling led to improved ovarian function. Basically, it is well known that PDGF is a growth factor that regulates cell growth and division and plays a role in blood vessel formation, angiogenesis and embryonic development. Previously, we examined whether PRL-1-overexpressing PD-MSCs secreted PDGF-BB by using Profiler Human XL Cytokine Arrays. We found that the PDGF-BB level in the supernatant was significantly increased in the Naïve group compared to the PRL-1 group (Supplementary Fig. 1a, b). Hence, we investigated the homology of PDGFBB between humans and rats in the $\mathrm{NIH}$ website (http://ncbi.nim.nih.gov), and the similarity was $86 \%$. In the serum of the OVX rats, the level of VEGF, a vascular remodeling factor, showed no difference among the groups (Fig. 2a). For this reason, the level of PDGF-BB, which is a PDGF ligand that plays a role in angiogenesis and pericyte recruitment, was significantly decreased in the NTx and Naïve Tx groups and substantially increased in the PRL-1 Tx groups compared to the Naïve Tx groups (Fig. $\left.1 \mathrm{e} ;{ }^{*} p<0.05\right)$. These data indicated that increased human PDGF-BB by transplanted PRL-1-overexpressing PD-MSCs had an angiogenic effect in the ovary tissues of OVX rats.

Additionally, to analyze the expression of PDGF-B and its receptors (e.g., PDGFRa and PDGFRß), we assessed the mRNA expression in ovarian tissues. The mRNA expression of PDGF-B was decreased in the NTx and Naïve Tx groups compared to the normal group and increased in the PRL-1 Tx groups compared to the NTx groups (Fig. 1f). Likewise, the mRNA expression of PDGFRa and PDGFR $\beta$ was higher in the PRL-1 Tx groups than in the NTx and Naïve groups (Fig. $1 \mathrm{~g}, \mathrm{~h} ;{ }^{*} p<0.05$ ). Moreover, the protein expression of PDGFRa and PDGFR $\beta$ was analyzed in ovarian tissues. The protein expression of PDGFRa was significantly increased in all groups compared to the normal group (Fig. 2b). Interestingly, the protein expression of PDGFR $\beta$ was significantly decreased in the NTx groups compared to the normal group and increased in the Naïve and PRL-1 Tx groups compared to the NTx groups at 1 week (Fig. $2 \mathrm{c} ;{ }^{*} p<0.05$ ). PDGFRa and PDGFR $\beta$ are known to regulate follicular development and vascular function, including angiogenesis and blood vessel formation in the ovary.

Hence, we analyzed the PDGF signaling pathway downstream [e.g., protein kinase C-delta (PKC $)$ ), protooncogene tyrosine protein kinase Src (Src), VEGF and VEGFR2] to determine whether the PDGF signaling pathway is activated by PRL-1-overexpressing cell transplantation in ovarian tissues of the OVX rats. The mRNA expression of PKCס, which interacts with PRL-1 and regulates proliferation and migration, was decreased in all groups compared to the normal group (Supplementary Fig. 2a). The positive signals of pSrc expression were higher in the PRL-1 Tx groups than in the NTx and Naïve Tx groups at 1 week (Supplementary Fig. 2b). Also, we analyzed the expression of the VEGF signaling pathway-induced PDGF gene in rat serum and the mRNA and protein levels in ovarian tissues. The mRNA expression of VEGF and VEGFR2 was significantly decreased in the PRL-1 Tx group compared to the NTx and Naïve Tx groups (Supplementary Fig. 2c, d). The protein expression of VEGFR2 was substantially decreased in the NTx groups compared to the normal group and increased in the Naïve and PRL-1 Tx groups compared to the NTx groups at 1 week and 3 weeks (Supplementary Fig. 2e). Interestingly, the protein expression of VEGFR2 in the ovary showed a similar pattern to the PDGFR $\beta$ gene expression in the ovaries of the OVX rats. Additionally, the mRNA expression of HIF1a and endoglin, which are proangiogenic factors and upstream of the VEGF signaling pathway, was significantly increased in the PRL-1 Tx groups compared to 
the NTx and Naïve Tx groups (Supplementary Fig. 3a, b; ${ }^{*} p<0.05$ ). The protein expression of endoglin in the ovary was similar to PDGFR $\beta$ protein expression and increased in the PRL-1 Tx group compared to the NTx and Naïve Tx group at 1 week. However, the protein expression of HIF1a in the ovary was not different (Supplementary Fig. 3c, d; ${ }^{*} p<0.05$ ).

However, analysis of angiogenic factors in homogenized whole ovaries is inappropriate for vascular remodeling studies. Hence, we performed immunohistochemical staining to determine whether PDGFR $\beta$ was expressed in mature follicles and arteries in ovarian tissues. As shown in Figure 2d, PDGFR $\beta$ was localized in antral follicles and arteries in ovarian tissues. To demonstrate the difference in each group, we analyzed the expression of PDGFR $\beta$ in follicles and arteries of ovarian tissues using the 3D HISTECH program. The results showed that PDGFR $\beta$ expression in antral follicles and arteries was significantly decreased in the NTx group compared to the normal group and substantially increased in the Naïve and PRL-1 Tx groups (Fig. 2e, f; ${ }^{\star} p<0.05$ ). These data indicated that the secreted PDGF from transplanted PRL-1-overexpressing PD-MSCs activates the PDGF signaling pathway in arteries and antral follicles of ovarian tissues.

\section{PRL-1-overexpressing PD-MSCs transplantation enhanced pericyte recruitment in the arteries of ovarian tissues via the PDGF signaling pathway.}

Pericyte coverage induced by PDGF, which is the main factor related to pericyte recruitment in blood vessels, is required for the stabilization of immature endothelial tubes [20,21]. Hence, we confirmed the expression of pericytes and endothelial cells through the ratio of neuron-glial antigen 2 (NG2) and cluster of differentiation 31 [CD31; platelet endothelial cell adhesion molecule (PECAM-1)] in ovarian tissues. As a result, the ratio of NG2 to CD31 in ovarian tissues was decreased in the NTx groups compared to the normal group and increased in the Naïve and PRL-1 Tx groups (Fig. 3a-c). To analyze the ratio of NG2 to CD31 in vessels of ovarian tissues (e.g., pericytes and endothelial cells), we performed immunofluorescence staining of ovarian tissues from the OVX rats. As shown in Figure $3 f$, the expression of NG2 and CD31 was localized in the pericytes and endothelium of vessels. To quantify positive signals, we analyzed the ratio between NG1 and CD31 expression in vessels using the ImageJ program. The ratio of NG2 to CD31 expression in vessels was significantly decreased in the NTx group compared to the normal group and increased in the PRL-1 group compared to the Naïve group (Fig. $3 \mathrm{~h} ;{ }^{*} p<0.05$ ). These data suggested that transplanted PRL-1-overexpressing PD-MSCs induced pericyte recruitment of vessels for vascular remodeling in the ovaries of the OVX rats.

Next, we analyzed autophagy during vascular regeneration in endothelial cells to demonstrate vessel stability by pericyte recruitment. To analyze vascular remodeling through the autophagic signaling pathway, we analyzed the expression of genes related to the autophagic signaling pathway (e.g., LC3B and $\mathrm{mTOR}$ ) in the ovarian tissues of the OVX rats (Fig. 3d). The protein expression of LC3B, which is an autophagic regulator, was significantly increased in the ovary in the PRL-1 Tx groups compared to the 
NTx and Naïve Tx groups (Fig. 3e; * $p<0.05$ ). To demonstrate LC3B expression in vessels for autophagosome formation, we performed immunofluorescence staining of ovarian tissues from the OVX rats. The expression of CD31 and LC3B was localized in vessels of ovarian tissues (Fig. $3 \mathrm{~g}$ ).

Quantification of LC3B-positive signals versus CD31-positive signals showed that the expression of LC3B in vessels was significantly decreased in the NTx groups compared to the normal group and substantially increased in the PRL-1 Tx groups compared to the NTx and Naïve Tx groups (Fig. $3 h ;{ }^{*} p<0.05$ ). These data indicated that transplanted PRL-1-overexpressing PD-MSCs induced the autophagic signaling pathway in the vessels of the ovaries of the OVX rats. Therefore, we confirmed the correlation between pericyte recruitment and autophagy (Fig. 3h). The constant value was 0.6040 , confirming this correlation. These data indicated that transplanted PRL-1-overexpressing PD-MSCs induced autophagy by pericyte recruitment in the vessels of the ovaries of the OVX rats.

\section{PRL-1-overexpressing PD-MSCs transplantation restored vascular remodeling by decreasing vascular permeability.}

To analyze the vascular function improvement mediated by vascular regeneration, we analyzed vascular permeability factors [e.g., early growth response 3 (erg-3)] in the ovaries of the OVX rats. The mRNA expression of erg-3, which is a transcription factor that regulates vascular permeability and vascular homeostasis, was increased in the PRL Tx groups compared to the NTx groups and the Naïve Tx groups (Fig. 4a; ${ }^{\star} p<0.05$ ). Additionally, the protein expression of erg-3 in isolated nuclei of ovaries was increased in the PRL Tx groups compared to the NTx groups and the Naïve Tx groups (Fig. 4b; ${ }^{\star} p<0.05$ ).

To demonstrate the effect of PRL-1 by focusing on endothelial cells, we performed in vitro experiments using HUVECs. After 5-fluorouracil (5-FU) treatment of HUVECs, Naïve and PRL-1-overexpressing cells were cocultured with HUVECs using insert systems for $48 \mathrm{~h}$. As shown in Figure 4c, the tube formation of the endothelial cells showed substantial disruption of bridges and branching points without coculture with HUVECs and strong organization of bridges and branching points in Naïve and PRL-1-overexpressing cells cocultured with HUVECs (Fig. 4c, d; * $p<0.05$ ). Moreover, to confirm the restoration of vascular function, we analyzed vascular permeability using a dextran assay at $48 \mathrm{~h}$. Vascular permeability was increased in the NTx group compared to the normal group and decreased in the Naïve and PRL-1 Tx groups compared to the NTx group (Fig. $4 \mathrm{e} ;{ }^{*} p<0.05$ ). These data indicated that transplanted PRL-1overexpressing PD-MSCs enhanced vascular function through an activated regulator of vascular permeability in the OVX rats.

\section{PRL-1-overexpressing PD-MSCs transplantation enhanced ovarian function through sex hormone levels and follicular development in an OVX rat model.}


In addition to regulating ovarian functions, the balance of endocrinal hormones is required for follicular development. To confirm the effect of PRL-1 on endocrinal hormones, we analyzed the levels of reproductive hormones, such as anti-Mullerian hormone (AMH), estradiol (E2), FSH and testosterone (TES), in the serum of the OVX rats using enzyme-linked immunosorbent assays (ELISAs). As a result, the levels of $\mathrm{AMH}$ and $\mathrm{E} 2$, which are involved in ovarian reserve and function, in individual serum samples were decreased in the NTx group compared to the normal group and significantly increased in the PRL-1 group compared to the NTx and Naïve Tx groups (Fig. 5a, b; ${ }^{*} p<0.05$ ). However, the level of FSH in individual serum samples was decreased in the NTx, Naïve and PRL-1 Tx groups compared to the normal group and showed no differences among the groups (Fig. $5 c ;{ }^{*} p<0.05$ ). In contrast, the level of TES in individual serum samples was increased in the NTx and Naïve Tx groups compared to the normal group and decreased in the PRL-1 Tx groups compared to the NTx groups (Fig. 5d). Additionally, we analyzed the factors related to follicular development in the ovaries of the OVX rats. As a result, the protein expression of the newborn ovary homeobox gene (Nobox), which is an oocyte-specific gene involved in early follicular development, was found to be increased in the ovaries of the OVX rats. In particular, the protein expression of Nobox was significantly increased in the PRL-1 Tx groups compared to the Naïve Tx groups (Fig. 5e; ${ }^{*} p<0.05$ ). Additionally, the protein expression of bone morphogenetic protein 15 (BMP15) and epidermal growth factor receptor (EGFR), which is involved in follicle maturation in late follicular development, was increased in the NTx groups compared to the normal group. In particular, the protein expression of BMP15 and EGFR was significantly increased in the PRL-1 Tx groups compared to the NTx and Naïve Tx groups (Fig. 5f, g; ${ }^{\star} p<0.05$ ).

To confirm the effect of PRL-1 on follicular development, we stained serially sectioned ovarian tissues from each group using H\&E and analyzed the samples using the 3D HISTECH program. Matured follicles were different in each group (Fig. 5h). To quantify the ovarian follicles according to maturation stage, we counted the follicles in the stained tissues. In the NTx groups, decreased primordial follicles and increased follicular atresia were observed compared to those in the normal groups. In the Naïve and PRL1 Tx groups, primordial follicles were recovered compared to those in the NTx group. Interestingly, follicular atresia was decreased in the PRL-1 Tx groups compared to the NTx and Naïve Tx groups. Unexpectedly, follicles in the other stages (e.g., primary, secondary and antral follicles) were not significantly different among the groups (Fig. 5i, Supplementary Table S2). These results indicated that transplanted PRL-1-overexpressing cells improved follicular development and hormone levels in the OVX rats.

\section{Effect of vascular remodeling and follicular development of the PRL-1 overexpressing PD-MSCs treated with siPRL-1 or PDGF inhibitor and recombinant PDGF-BB ex vivo.}

To analyze the effect of PRL-1-overexpressing PD-MSCs transplantation in the ovaries of the OVX rats, we performed an ex vivo experiment using siRNA-PRL-1. We analyzed the mRNA expression of the human- 
specific Alu (hAlu) sequence in the injected ovaries to confirm the engraftment activity of human PDMSCs. The mRNA expression of the hAlu sequence was strongly increased in the Naïve and PRL-1 injection groups. However, the mRNA expression of the hAlu sequence was significantly decreased in the siRNA-PRL-1 injection groups compared to the Naïve and PRL-1 injection groups (Fig. 6a; ${ }^{*} p<0.05$ ). We analyzed the mRNA expression of human PRL-1 in ovarian tissues to confirm the expression of injected PRL-1. Interestingly, the mRNA expression of human PRL-1 was at the basal level in the Naïve injection groups. The mRNA expression of human PRL-1 was substantially increased in the PRL-1 injection groups compared to the control and Naïve injection groups. However, the mRNA expression of human PRL-1 was significantly decreased in the siRNA-PRL-1 injection groups compared to the PRL-1 injection groups (Fig. $\left.6 \mathrm{~b} ;{ }^{\star} p<0.05\right)$. Additionally, we analyzed the mRNA expression of the PDGF family in ovarian tissues. The mRNA expression of PDGF-BB, which is a classic PDGF ligand related to vascular remodeling, was significantly increased in the Naïve and PRL-1 injection groups compared to the control groups. The mRNA expression of PDGF-BB was decreased in the siRNA-PRL-1 group compared to the Naîve and PRL1 injection groups (Fig. $6 \mathrm{c} ;{ }^{*} p<0.05$ ). The mRNA expression of PDGFR $\beta$, which is a representative PDGF receptor related to vascular remodeling, was strongly increased in the Naïve and PRL-1 injection groups compared to the control groups. The mRNA expression of PDGFR $\beta$ was significantly decreased in the siRNA-PRL-1 injection groups compared to the Naïve and PRL-1 injection groups (Fig. $6 \mathrm{~d} ;{ }^{*} p<0.05$ ).

To analyze the effect of PDGF via transplantation of PRL-1-overexpressing PD-MSCs in the ovaries of the OVX rats, we performed an ex vivo experiment using imatinib as a PDGFR inhibitor and recombinant PDGF-BB. We analyzed the mRNA expression of human PRL-1 in ovarian tissues to confirm the expression of cocultivated PRL-1-overexpressing cells. The groups with PRL-1-overexpressing PD-MSCs and PDGFR inhibitor showed increased mRNA expression of human PRL-1 compared to the PRL-1 groups (Fig. 6e; ${ }^{*} p<0.05$ ). These data indicated that PRL-1 affects PDGF signaling, whereas PDGF signaling does not affect PRL-1. We analyzed the mRNA expression of the PDGF family in cocultivated ovaries. The mRNA expression of PDGF-BB was significantly increased in the Naïve and PRL-1 cocultivation groups compared to the control and recombinant PDGF-BB + Tx groups. The cocultivated groups with Naïve and PRL-1-overexpressing PD-MSCs treated with PDGF receptor inhibitor showed significantly decreased mRNA expression of PDGF-BB compared to the Naïve and PRL-1 cocultivation groups (Fig. $6 f ;{ }^{*} p<0.05$ ). The mRNA expression of PDGFR $\beta$ was significantly increased in the Naïve and PRL-1 cocultivation groups compared to the control and recombinant PDGF-BB Tx groups. Compared to PRL-1overexpressing PD-MSC cocultivation, cocultivation with PRL-1-overexpressing PD-MSCs treated with PDGF receptor inhibitor significantly decreased the mRNA expression of PDGFRß (Fig. $6 \mathrm{~g} ;{ }^{*} p<0.05$ ). In addition, we analyzed VEGF signaling in vascular remodeling in cocultivated di-sectioned ovaries. The mRNA expression of VEGF was decreased in the Naïve and PRL-1 groups compared to the recombinant PDGF-BB cocultivation groups. The mRNA expression of VEGF was significantly decreased in the Naïve and PRL-1 cocultivation groups compared to the Naïve and PRL-1 cocultivation groups (Fig. 6h; ${ }^{*}<0.05$ ). The mRNA expression of VEGFR2 showed no difference among the groups excluding the control group (data not shown). These results indicate that VEGF binds PDGF receptors, but VEGFR2 is a different signaling pathway from the PDGF signaling pathway [22]. 


\section{Discussion}

Vascular remodeling is essential and important for the regeneration of damaged organs as well as organogenesis. This process plays a role in delivering oxygen as well as various nutrients that (1) enlarge the vascular network in ischemic tissues for therapeutic purposes by angiogenesis and (2) invade the vascular network to enable progenitor survival and differentiation [23]. Although vascular structure and blood flow are estrous cycle dependent in reproductive organs including uterus, but their changes in ovarian tissues are limited [24]. For the treatment of degenerative diseases, stem cells have been researched for their therapeutic mechanisms, and their paracrine effects were reported to be mediated by the secretion of proangiogenic factors such as PDGF, VEGF and FGFs [25, 26]. Moreover, MSC-based treatment of damaged tissues could enhance microvascular density with increasing tissue perfusion. Hence, various paracrine effects of MSCs can enhance vascular remodeling in infarction [27]. In particular, abnormalities in ovarian angiogenesis were observed in patients with PCOS. PCOS is a type of ovarian dysfunction with pathological characteristics, and clinical trials of various drugs for treatment of PCOS have been performed $[28,29]$.

Recently, Cho et al. and colleagues demonstrated that transplanted PD-MSCs improve ovarian function via the VEGF pathway in OVX rats [11]. However, there is a lack of research on the regenerative mechanism involved in vascular regeneration, and MSCs have a weaker mode of action (MOA) in improving ovarian function through vascular remodeling. To overcome these issues, many researchers have studied functionally enhanced stem cells for degenerative diseases [30, 31]. However, the therapeutic mechanism of functionally enhanced stem cells in rats with ovarian dysfunction has never been reported until now. In our study, we used functionally enhanced PD-MSCs for the treatment of ovarian dysfunction and to maximize their therapeutic efficacies in ovarian dysfunction model. Finally, we estimated the therapeutic mechanisms of PRL-1 on rats with ovarian dysfunction by stimulating vascular regeneration.

PRLs, including PRL-1, PRL-2 and PRL-3, are involved in cell migration together with differentiation for liver regeneration. Recently, these molecules were shown to be involved in the regulation of endothelial cell differentiation in the digestive system [32,33]. Additionally, Kim et al. reported that PRL-1-enhanced PD-MSCs induced liver regeneration by vascular remodeling through alterative expressions of various miRNAs and targeted genes. The researchers found a slight correlation in which the expression of PDGFRa and PDGFR $\beta$ was increased in the PRL-1-enhanced PD-MSCs Tx groups compared to the PDMSCs Tx groups [34]. Otherwise, researchers reported that PRL-1 inhibited endothelial cell differentiation by reducing the expression of adhesion molecules in inflammation-induced endothelial cells in cancer although their correlation between PRL-1 and PDGF still unclear on vascular remodeling [35]. Therefore, our data suggested that their correlation between PRL-1 and PDGF could be used as a fundamental evidence of vascular remodeling processing in order to restore injured tissues including ovary. Recent reports found higher PRL-1, PRL-2 and PRL-3 transcript levels in the vasculature and endothelial cells. Blockade of PRL-2 inhibited vascular remodeling by suppressing endothelial cell migration via the VEGFA/NOTCH-1 signaling pathway in the postnatal mouse retina [15]. Also, Xu et al. demonstrated that PRL-3 
correlated with VEGF in HUVECs downstream of VEGF/MEF2C [14]. Based on these reports, we focused not only on whether the paracrine effect of PRL-1 regulates vascular function and ovarian function but also on which factor mediated by PRL-1 is a major regulator in the OVX rats.

During vascular development, pericytes collaborate with endothelial cells and are major regulators of vascular remodeling, including stabilization and maturation. Specifically, these cells modulate the metastasis of endothelial cells (e.g., cell growth, proliferation, differentiation and migration) and smooth muscle cell contraction for capillary blood flow for vascular remodeling [36]. Our findings indicated that transplanted PD-MSCs overexpressing PRL-1 induced the expression of NG2, which is related to pericyte recruitment, and erg-3, which is related to vascular permeability, in the arteries of ovarian tissues.

Additionally, several researchers have reported that PRL-1 is correlated with reproductive systems. PRL-1 is localized in the surface epithelium and oviduct of the ovary. Specifically, higher expression of PRL-1 was shown in the stroma, granulosa cells, corpus luteum, and vasculature with the medulla [37]. Several studies have reported that PRL-1 is regulated by FSH, and we also confirmed these results by demonstrating that transplanted PRL-1-overexpressing cells improved ovarian function in the OVX rats $[13,38]$.

In young adult age, ovariectomy affects alters follicle development but maintains ovarian function because increases atresia follicle but also increases compensatory antral follicle [39]. As a result, transplanted PRL-1-overexpressing PD-MSCs enhanced the concentration of AMH and estrogen as well as the expression of genes related to follicular development in the OVX rats. The follicles were counted, and PRL-1 resulted in increased primordial follicles and decreased follicular atresia in the OVX rats. Transplanted PRL-1-overexpressing PD-MSCs improved ovarian function through vascular remodeling compared to NTx group. However, the further study on the paracrine effect of PRL-1-enhanced PD-MSCs on vascular remodeling and ovarian dysfunction should be needed.

Basically, PDGF, as a proangiogenic factor, plays a role in vascular remodeling and steroid production for ovarian function, and it leads to mature and stable endothelial cells [20,40]. So, we confirmed that PDGFBB increased by PRL-1-enhanced PD-MSCs bind with their receptors and mediate the PDGF signaling pathway in cytokine array analysis and the expression of PDGFR $\beta$ in arteries and follicles of ovarian tissues. As shown in Fig. 2d, the expression and localization of PDGF were confirmed in arteries specific to the permeable theca layer of follicles in ovarian tissues and were increased in the PRL-1 group compared to the Naiive group. Despite these results, PDGF secreted by PRL-1-enhanced PD-MSCs showed higher expression in the arteries and theca layers of follicles. Based on previous results, we analyzed the correlation between PRL-1 and PDGF in the ovary to confirm the paracrine effect of PRL-1. In our ex vivo data, the lower level of PRL-1 after siRNA treatment inhibited the expression of PDGFR $\beta$ in the ovary. The expression of factors related to follicular development in ovarian tissues was decreased, and PRL-1 expression was suppressed in PD-MSCs by siRNA. In contrast, PDGF in PD-MSCs treated with recombinant PDGF or an inhibitor regulated the expression of PDGF-BB and PDGFR $\beta$ and the VEGF pathway in ovarian tissues. Therefore, our data suggested the paracrine effect of PRL-1 could be restored 
ovarian function in the OVX rats through activation of PDGF signaling pathway by their correlation between PRL-1 and PDGF.

In conclusion, our findings indicated that PRL-1-enhanced PD-MSCs regulated the vascular formation of HUVECs and secreted higher levels of PDGF than PD-MSCs in vitro. After PRL-1-enhanced PD-MSC transplantation into the OVX rats, vascular remodeling and pericyte recruitment were enhanced, along with the autophagic pathway. Moreover, transplanted PRL-1-enhanced PD-MSCs restored ovarian function, including reproductive hormone levels and follicular development, in the OVX rats. Therefore, these findings offer new insights into functionally enhanced stem cell therapy for reproductive medicines.

\section{Conclusion}

In conclusion, PRL-1 restore ovarian function by enhanced vascular function through PDGF signaling pathway (Fig. 7). These findings offer new insight into the effects of functionally enhanced stem cell therapy for reproductive systems and should provide new avenues to develop more efficient therapies in degenerative medicine.

\section{Abbreviations}

POF: premature ovarian failure; PCOS: polycystic ovary syndrome; HRT: hormone replacement therapy; MOA: mode of action; OVX: ovariectomy; PD-MSCs: placenta-derived mesenchymal stem cells; PRL-1 : phosphatase of regenerating liver-1, PD-MSCs ${ }^{\text {PRL-1; }}$ hAlu: human specific Alu; PDGF: platelet-derived growth factor; VEGF: vascular endothelial growth factor; FGF: fibroblast growth factor; H\&E staining: hematoxylin and eosin staining; ELISAs: enzyme-linked immunosorbent assays; NTx: non-transplantation; PKCס: protein kinase C-delta; Src: proto-oncogene tyrosine-protein kinase Src; CD31: platelet endothelial cell adhesion molecule (PECAM-1); NG2: neuron-glial antigen 2; mTOR: mammalian target of rapamycin; LC3B: microtube-associated protein 1 light chain 3; GAPDH: glyceraldehyde 3-phosphate dehydrogenase; HUVECs: human umbilical vein endothelial cells; 5FU: 5-Fluorouracil; AMH: anti-Mullerian hormone; E2: estrogen; FSH: follicle stimulating hormone; TES: testosterone; Nobox: newborn ovary homeobox; BMP15: bone morphogenetic protein 15; EGFR: epidermal growth factor receptor

\section{Declarations}

\section{Acknowledgments}

The authors would like to thank Ms. Ha Eun Shin (University of CHA, Gyeonggi-do, Korea) for technical supporting, respectively.

\section{Author contributions}


Conceptualization, G.J.K.; Animal model construction, validation and formal analysis, H.P., J.S., J.H.Y., ; Cell culture, H.P. and J.Y.K.; Image data analysis, J.Y.L.; Writing and editing, H.P., J.S. and G.J.K.; project administration, and funding acquisition, G.J.K.. All authors have read and agreed to the published version of the manuscript.

\section{Author information}

Hyeri Park, MS, ${ }^{1}$ Department of Biomedical Science, CHA University, 335 Pangyo-Ro, Bundang-Gu, Seongnam-si, Gyeonggi-Do, 13488, Republic of Korea; Tel: 82-31-881-7145; Fax: 82-31-881-4102; email: hyeyeyeri@gmail.com; http://orcid.org/0000-0003-2274-5313

Jin Seok, PhD, ${ }^{1}$ Department of Biomedical Science, CHA University, 335 Pangyo-Ro, Bundang-Gu, Seongnam-si, Gyeonggi-Do, 13488 , Republic of Korea; ${ }^{2}$ Research institute of Placenta Science, CHA University, Seongnam-si, Gyeonggi-do, 13488, Republic of Korea; Tel: 82-31-881-7145; Fax: 82-31-8814102; e-mail: jjin8977@gmail.com; http://orcid.org/0000-0001-5805-3736

Jun Hyeong You, MS, ${ }^{1}$ Department of Biomedical Science, CHA University, 335 Pangyo-Ro, Bundang-Gu, Seongnam-si, Gyeonggi-Do, 13488, Republic of Korea; Tel: 82-31-881-7145; Fax: 82-31-881-4102; email: yjh950210@gmail.com; http://orcid.org/0000-0001-6436-6077

Jae Yeon Kim, PhD, ${ }^{1}$ Department of Biomedical Science, CHA University, 335 Pangyo-Ro, Bundang-Gu, Seongnam-si, Gyeonggi-Do, 13488, Republic of Korea; ${ }^{2}$ Research institute of Placenta Science, CHA University, Seongnam-si, Gyeonggi-do, 13488, Republic of Korea; Tel: 82-31-881-7145; Fax: 82-31-8814102; e-mail:janejykim92@gmail.com; http://orcid.org/0000-0002-3457-5190

Ja-Yun Lim, PhD, ${ }^{3}$ Department of Health and Environmental Science, Korea University, 145 Anam-Ro, Seongbuk-Gu, Seoul, 02481, Republic of Korea; email: jayun78@korea.ac.kr; http://orcid.org/0000-00015874-8765

Gi Jin Kim, PhD, ${ }^{1}$ Department of Biomedical Science, CHA University, 689, Sampyeong-dong, Bundang-gu, Seongnam-si, Gyeonggi-do, Republic of Korea; ${ }^{2}$ Research institute of Placenta Science, CHA University, Seongnam-si, Gyeonggi-do, 13488, Republic of Korea; Tel: 82-31-881-7145, Fax: 82-31-881-4102, e-mail: gjkim@cha.ac.kr; http://orcid.org/0000-0002-2320-7157

\section{Ethics approval and consent to participate}

All animal experiments were approved by the Institutional Animal Care and Use committee (IACUC 190048) of the CHA Laboratory Animal Research Center at Sampyeong-dong in Gyeonggi, Republic of Korea. Also, the collection of human placenta and their use were conducted under the guidelines and with 
the approval of the affiliated Institutional Review Board of the Gangnam CHA General Hospital, Seoul, Korea (IRB 07-18). All patients provided written informed consent to the respective use of their tissues.

\section{Consent for publication}

The authors declare that there has no conflict of interest.

\section{Data availability statement}

The data that support the findings of this study are available from the corresponding author upon reasonable request.

\section{Funding}

This work was supported by the Basic Science Research Program through the National Research Foundation of Korea (NRF) funded by the Ministry of Science, ICT \& Future Planning (2017M3A9B4061665).

\section{Supporting information}

Additional supporting information may be found online in the supporting information section.

\section{References}

1. Devesa J, Caicedo D. The Role of Growth Hormone on Ovarian Functioning and Ovarian Angiogenesis. Front Endocrinol (Lausanne). 2019;10:450.

2. Jabbour HN. Vascular function in female reproduction. Reproduction. 2009;138(6):867-8.

3. Robinson RS, Woad KJ, Hammond AJ, Laird M, Hunter MG, Mann GE. Angiogenesis and vascular function in the ovary. Reproduction. 2009;138(6):869-81.

4. Heldin $\mathrm{CH}$, Westermark $\mathrm{B}$. Mechanism of action and in vivo role of platelet-derived growth factor. Physiol Rev. 1999;79(4):1283-316.

5. Nilsson EE, Detzel C, Skinner MK. Platelet-derived growth factor modulates the primordial to primary follicle transition. Reproduction. 2006;131(6):1007-15.

6. Ravn SH, Rosenberg J, Bostofte E. Postmenopausal hormone replacement therapy-clinical implications. Eur J Obstet Gynecol Reprod Biol. 1994;53(2):81-93.

7. Webber $L$, Anderson RA, Davies M, Janse F, Vermeulen N. HRT for women with premature ovarian insufficiency: a comprehensive review. Hum Reprod Open. 2017;2017(2):hox007. 
8. Yang Z, Du X, Wang C, Zhang J, Liu C, Li Y, Jiang H. Therapeutic effects of human umbilical cord mesenchymal stem cell-derived microvesicles on premature ovarian insufficiency in mice. Stem Cell Res Ther. 2019;10(1):250.

9. Santos RA, Asensi KD, de Barros JHO, de Menezes RCS, Cordeiro IR, Neto JMB, Kasai-Brunswick TH, Goldenberg R. Intrinsic Angiogenic Potential and Migration Capacity of Human Mesenchymal Stromal Cells Derived from Menstrual Blood and Bone Marrow. Int J Mol Sci. 2020;21(24).

10. Na J, Song J, Kim HH, Seok J, Kim JY, Jun JH, Kim GJ. Human placenta-derived mesenchymal stem cells trigger repair system in TAA-injured rat model via antioxidant effect. Aging. 2020;13(1):61-76.

11. Cho J, Kim TH, Seok J, Jun JH, Park H, Kweon M, Lim JY, Kim GJ. Vascular remodeling by placentaderived mesenchymal stem cells restores ovarian function in ovariectomized rat model via the VEGF pathway. Lab Invest. 2021;101(3):304-17.

12. Rios P, Li X, Kohn M. Molecular mechanisms of the PRL phosphatases. FEBS J. 2013;280(2):505-24.

13. Schmidt J, de Avila J, McLean D. Regulation of protein tyrosine phosphatase 4a1, B-cell translocation gene 2, nuclear receptor subfamily 4a1 and diacylglycerol 0-acyltransferase 1 by follicle stimulating hormone in the rat ovary. Reprod Fertil Dev. 2006;18(7):757-65.

14. Xu J, Cao S, Wang L, Xu R, Chen G, Xu Q. VEGF promotes the transcription of the human PRL-3 gene in HUVEC through transcription factor MEF2C. PLoS One. 2011;6(11):e27165.

15. Poulet M, Sirois J, Boye K, Uetani N, Hardy S, Daubon T, Dubrac A, Tremblay ML, Bikfalvi A. PRL-2 phosphatase is required for vascular morphogenesis and angiogenic signaling. Commun Biol. 2020;3(1):603.

16. Lee MJ, Jung J, Na KH, Moon JS, Lee HJ, Kim JH, Kim GI, Kwon SW, Hwang SG, Kim GJ. Anti-fibrotic effect of chorionic plate-derived mesenchymal stem cells isolated from human placenta in a rat model of CCl(4)-injured liver: potential application to the treatment of hepatic diseases. J Cell Biochem. 2010;111(6):1453-63.

17. Kim JY, Choi JH, Kim SH, Park H, Lee D, Kim GJ. Efficacy of Gene Modification in Placenta-Derived Mesenchymal Stem Cells Based on Nonviral Electroporation. Int J Stem Cells. 2021;14(1):112-8.

18. Myers M, Britt KL, Wreford NG, Ebling FJ, Kerr JB. Methods for quantifying follicular numbers within the mouse ovary. Reproduction. 2004;127(5):569-80.

19. Brown HM, Russell DL. Blood and lymphatic vasculature in the ovary: development, function and disease. Hum Reprod Update. 2014;20(1):29-39.

20. Zhang J, Cao R, Zhang Y, Jia T, Cao Y, Wahlberg E. Differential roles of PDGFR-alpha and PDGFRbeta in angiogenesis and vessel stability. FASEB J. 2009;23(1):153-63.

21. Tsioumpekou M, Cunha SI, Ma H, Ahgren A, Cedervall J, Olsson AK, Heldin $\mathrm{CH}$, Lennartsson J. Specific targeting of PDGFRbeta in the stroma inhibits growth and angiogenesis in tumors with high PDGF-BB expression. Theranostics. 2020;10(3):1122-35.

22. Mamer SB, Chen S, Weddell JC, Palasz A, Wittenkeller A, Kumar M, Imoukhuede PI. Discovery of HighAffinity PDGF-VEGFR Interactions: Redefining RTK Dynamics. Sci Rep. 2017;7(1):16439. 
23. Gianni-Barrera R, Di Maggio N, Melly L, Burger MG, Mujagic E, Gurke L, Schaefer DJ, Banfi A. Therapeutic vascularization in regenerative medicine. Stem Cells Transl Med. 2020;9(4):433-44.

24. Jokubkiene L, Sladkevicius P, Rovas L, Valentin L. Assessment of changes in volume and vascularity of the ovaries during the normal menstrual cycle using three-dimensional power Doppler ultrasound. Hum Reprod. 2006;21(10):2661-8.

25. Du W, Li X, Chi Y, Ma F, Li Z, Yang S, Song B, Cui J, Ma T, Li J, et al. VCAM-1 + placenta chorionic villiderived mesenchymal stem cells display potent pro-angiogenic activity. Stem Cell Res Ther. 2016;7:49.

26. Chen CY, Liu SH, Chen CY, Chen PC, Chen CP. Human placenta-derived multipotent mesenchymal stromal cells involved in placental angiogenesis via the PDGF-BB and STAT3 pathways. Biol Reprod. 2015;93(4):103.

27. Timmers L, Lim SK, Hoefer IE, Arslan F, Lai RC, van Oorschot AA, Goumans MJ, Strijder C, Sze SK, Choo A, et al. Human mesenchymal stem cell-conditioned medium improves cardiac function following myocardial infarction. Stem Cell Res. 2011;6(3):206-14.

28. Stouffer RL, Martinez-Chequer JC, Molskness TA, Xu F, Hazzard TM. Regulation and action of angiogenic factors in the primate ovary. Arch Med Res. 2001;32(6):567-75.

29. Irani M, Seifer DB, Grazi RV, Julka N, Bhatt D, Kalgi B, Irani S, Tal O, Lambert-Messerlian G, Tal R. Vitamin D Supplementation Decreases TGF-beta1 Bioavailability in PCOS: A Randomized PlaceboControlled Trial. J Clin Endocrinol Metab. 2015;100(11):4307-14.

30. Park JS, Suryaprakash S, Lao YH, Leong KW. Engineering mesenchymal stem cells for regenerative medicine and drug delivery. Methods. 2015;84:3-16.

31. Ye Z, Lu W, Liang L, Tang M, Wang Y, Li Z, Zeng H, Wang A, Lin M, Huang L, et al. Mesenchymal stem cells overexpressing hepatocyte nuclear factor-4 alpha alleviate liver injury by modulating antiinflammatory functions in mice. Stem Cell Res Ther. 2019;10(1):149.

32. Diamond RH, Peters C, Jung SP, Greenbaum LE, Haber BA, Silberg DG, Traber PG, Taub R. Expression of PRL-1 nuclear PTPase is associated with proliferation in liver but with differentiation in intestine. Am J Physiol. 1996;271(1 Pt 1):G121-9.

33. Kong W, Swain GP, Li S, Diamond RH. PRL-1 PTPase expression is developmentally regulated with tissue-specific patterns in epithelial tissues. Am J Physiol Gastrointest Liver Physiol. 2000;279(3):G613-21.

34. Kim JY, Jun JH, Park SY, Yang SW, Bae SH, Kim GJ. Dynamic Regulation of miRNA Expression by Functionally Enhanced Placental Mesenchymal Stem Cells PromotesHepatic Regeneration in a Rat Model with Bile Duct Ligation. Int J Mol Sci. 2019;20(21).

35. Sun JP, Wang WQ, Yang H, Liu S, Liang F, Fedorov AA, Almo SC, Zhang ZY. Structure and biochemical properties of PRL-1, a phosphatase implicated in cell growth, differentiation, and tumor invasion. Biochemistry. 2005;44(36):12009-21.

36. Bordenave J, Tu L, Berrebeh N, Thuillet R, Cumont A, Le Vely B, Fadel E, Nadaud S, Savale L, Humbert $\mathrm{M}$, et al. Lineage Tracing Reveals the Dynamic Contribution of Pericytes to the Blood Vessel 
Remodeling in Pulmonary Hypertension. Arterioscler Thromb Vasc Biol. 2020;40(3):766-82.

37. Dumaual CM, Sandusky GE, Crowell PL, Randall SK. Cellular localization of PRL-1 and PRL-2 gene expression in normal adult human tissues. J Histochem Cytochem. 2006;54(12):1401-12.

38. McLean DJ, Friel PJ, Pouchnik D, Griswold MD. Oligonucleotide microarray analysis of gene expression in follicle-stimulating hormone-treated rat Sertoli cells. Mol Endocrinol.

2002;16(12):2780-92.

39. Gosden RG, Telfer E, Faddy MJ, Brook DJ. Ovarian cyclicity and follicular recruitment in unilaterally ovariectomized mice. J Reprod Fertil. 1989;87(1):257-64.

40. Raza A, Franklin MJ, Dudek AZ. Pericytes and vessel maturation during tumor angiogenesis and metastasis. Am J Hematol. 2010;85(8):593-8.

\section{Figures}

Figure. 1
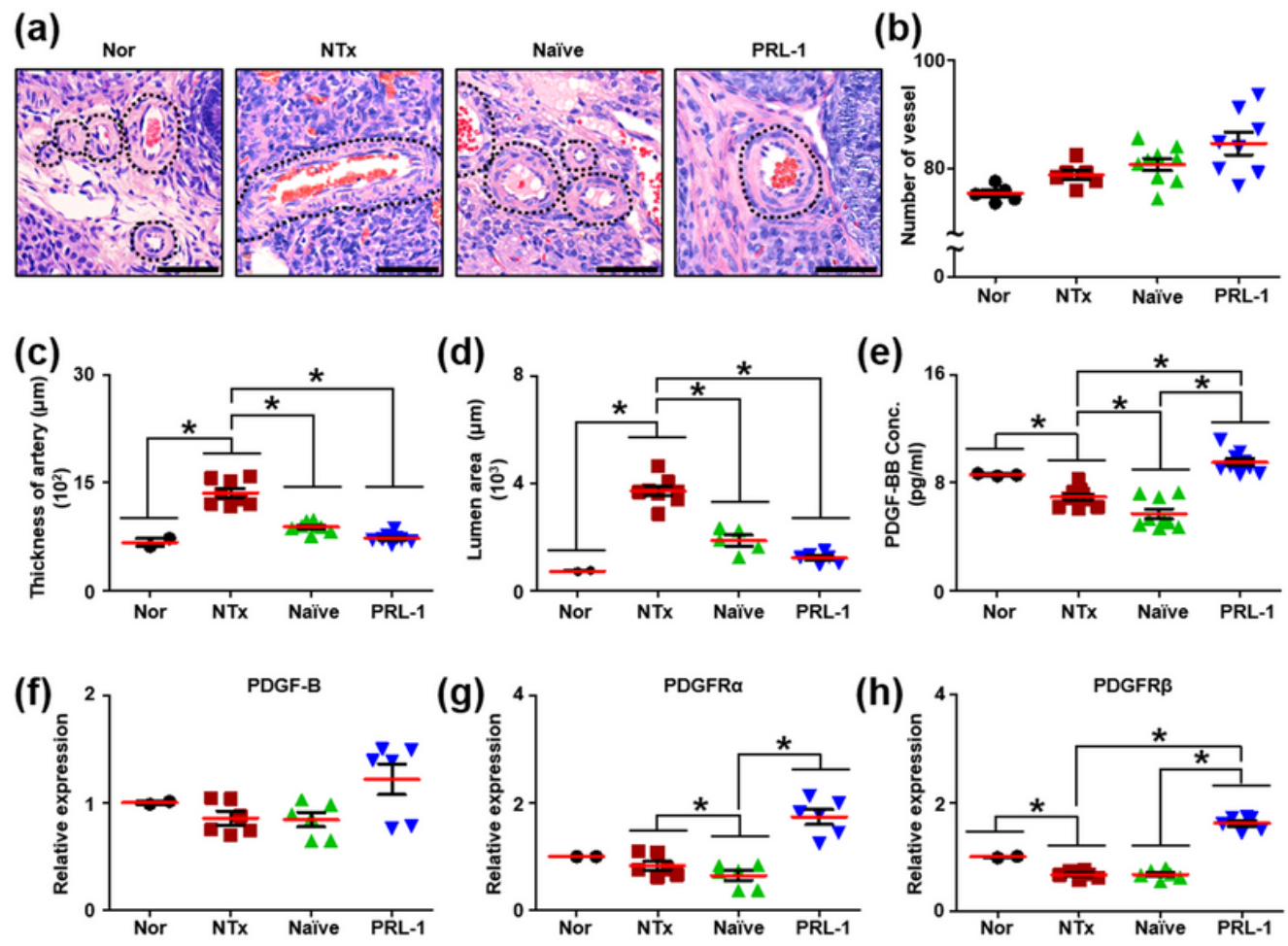

\section{Figure 1}

Effect of transplanted PRL-1 on vessels in the ovaries of OVX rats. (a) Histological analysis of the blood vessel structure in ovarian tissues was analyzed by H\&E staining. Dotted line: blood vessels. Scale bar: 
$1.5 \mathrm{~mm}$. Magnification: 200X. (b) The number of vessels, (c) thickness of the artery and (d) area of the lumen were analyzed by the 3D HISTECH program. (e) The concentration of PDGF-BB in serum was analyzed by ELISAs. (f) The mRNA expression of PDGF-B, (g) PDGFRa and (h) PDGFR 3 was analyzed by qRT-PCR. The data are representative of three independent experiments and expressed as the mean \pm S.D. * $p<0.05$, normal vs. NTx, NTx vs. Tx (Naïve and PRL-1), and Naïve vs. PRL-1 at each time point.

\section{Figure. 2}
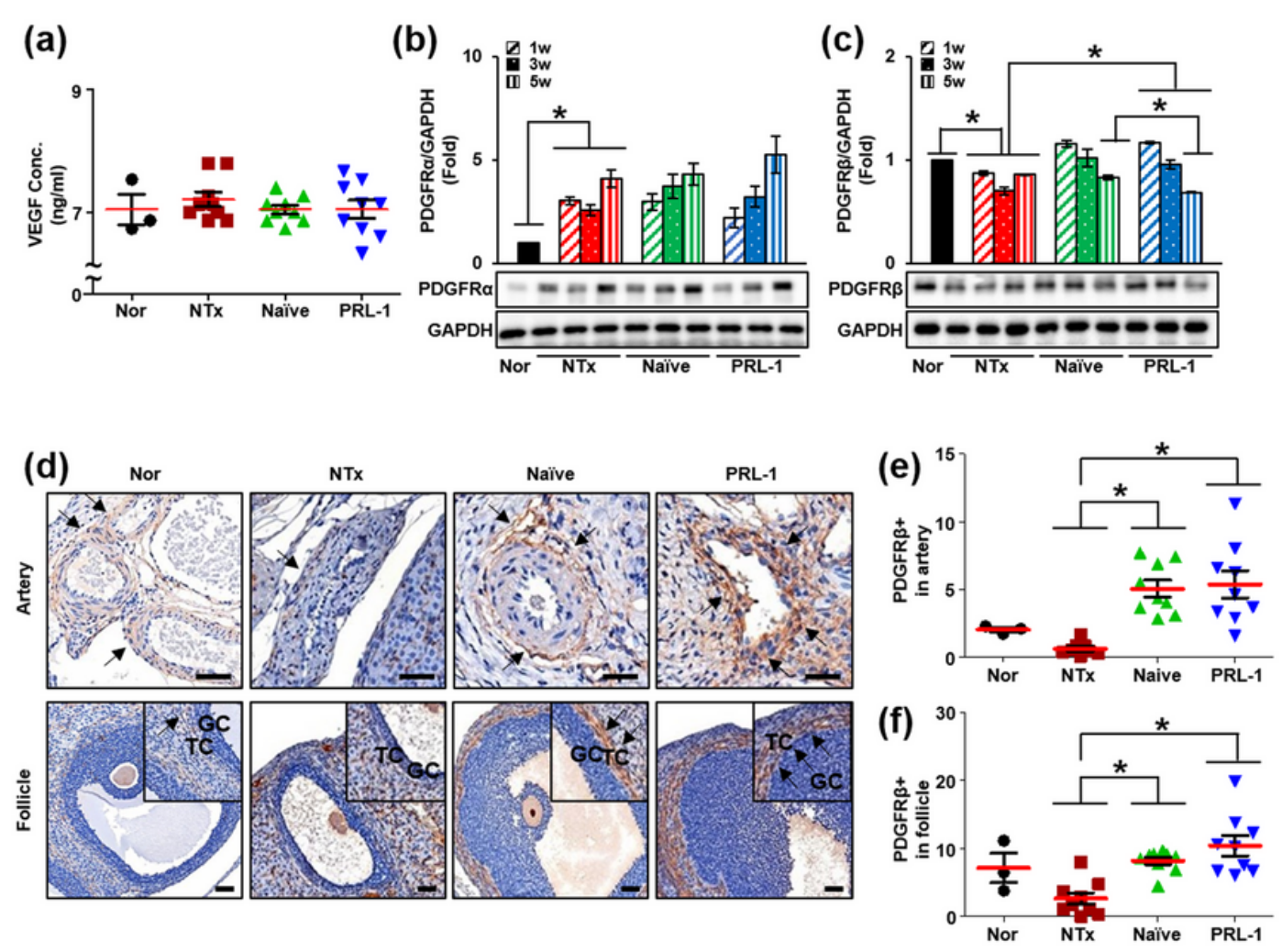

Figure 2

Effect of transplanted PRL-1-overexpressing cells on the PDGF signaling pathway in the ovaries of OVX rats. (a) The concentration of VEGF in serum was analyzed by ELISAs. (b) The protein expression of PDGFRa and (c) PDGFR $\beta$ was analyzed by western blots. (d) The localization and gene expression of PDGFR $\beta$ in arteries and follicles of ovarian tissues were analyzed by immunohistochemistry staining. (e) The PDGFR $\beta$ intensity of arteries and ( $f$ ) mature follicles in the ovary was analyzed by the 3D HISTECH program. The data are representative of three independent experiments and expressed as the means \pm S.D. * $p<0.05$, normal vs. NTx, NTx vs. Tx (Naïve and PRL-1), and Naïve vs. PRL-1 at each time point. 
Figure. 3
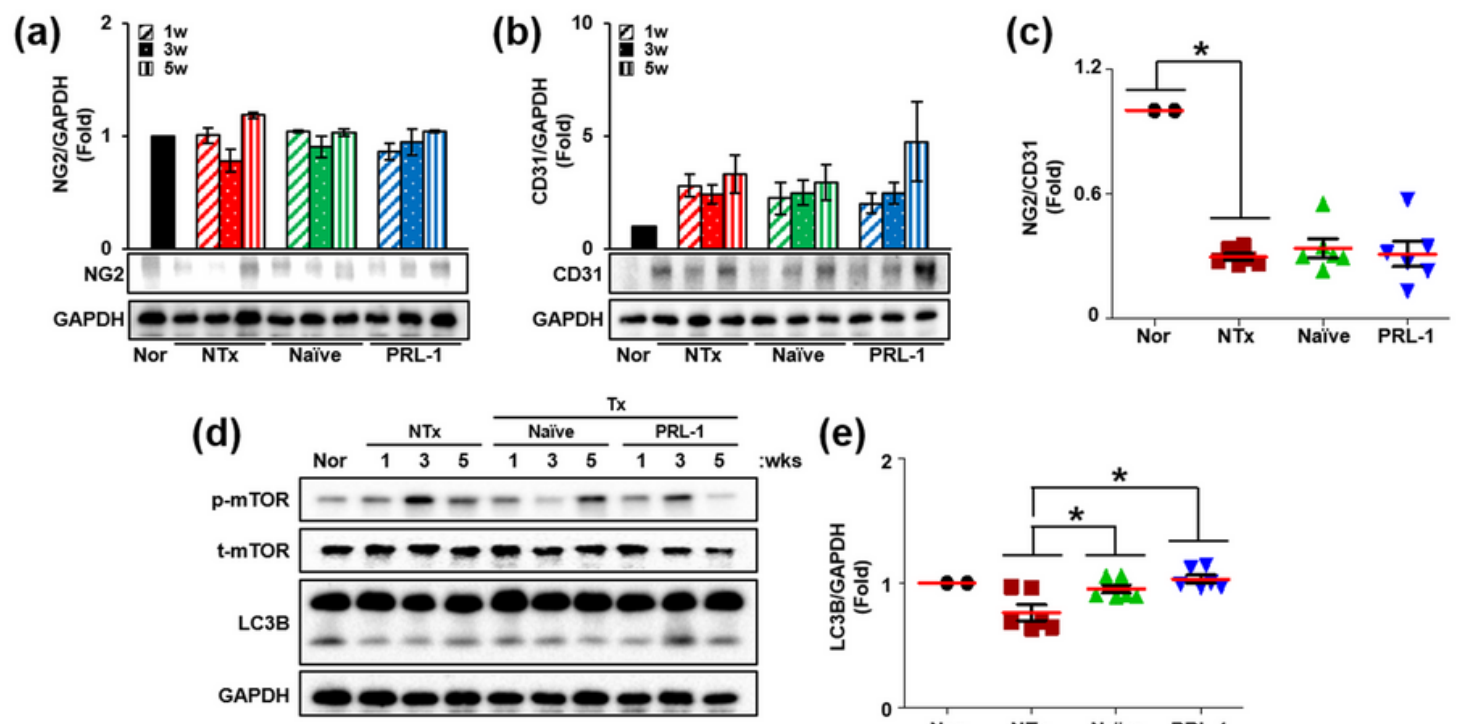

(e)
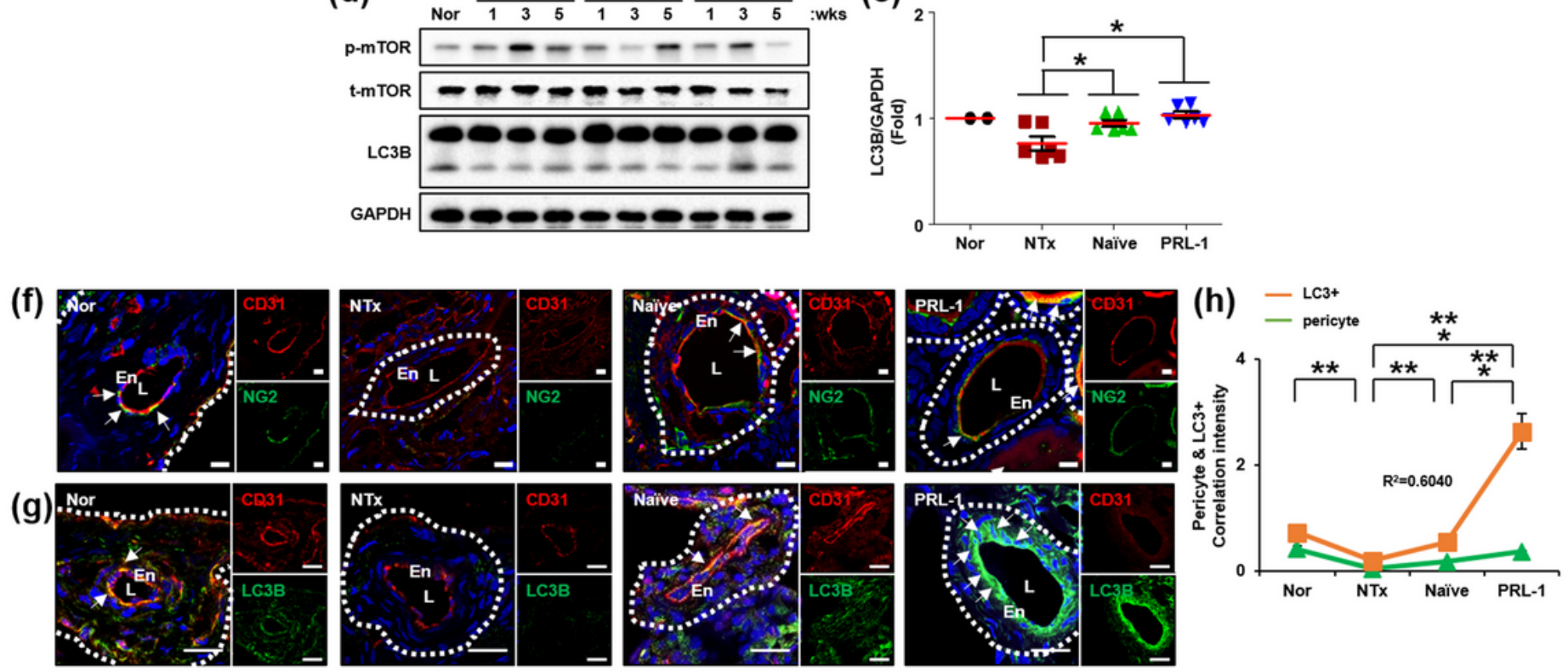

Figure 3

Effect of transplanted PRL-1-overexpressig cells on pericyte recruitment in the ovaries of OVX rats. (a) The protein expression of NG2 and (b) CD31 and (c) the ratio of NG2/CD31 were analyzed by western blots. (d) The gene expression of mTOR and LC3B and (e) the intensity of LC3B gene expression were analyzed by western blots and the ImageJ program. (f) The gene expression and localization of NG2 and CD31 and (g) LC3B and CD31 in arteries of ovarian tissues were analyzed by immunofluorescence staining. (h) The gene correlation by fluorescence intensity of NG2 and LC3B was analyzed by the ImageJ program. The data are representative of three independent experiments and expressed as the mean \pm S.D. * $p<0.05$, normal vs. NTx, NTx vs. Tx (Naïve and PRL-1), and Naïve vs. PRL-1 at each time point. 
Figure. 4
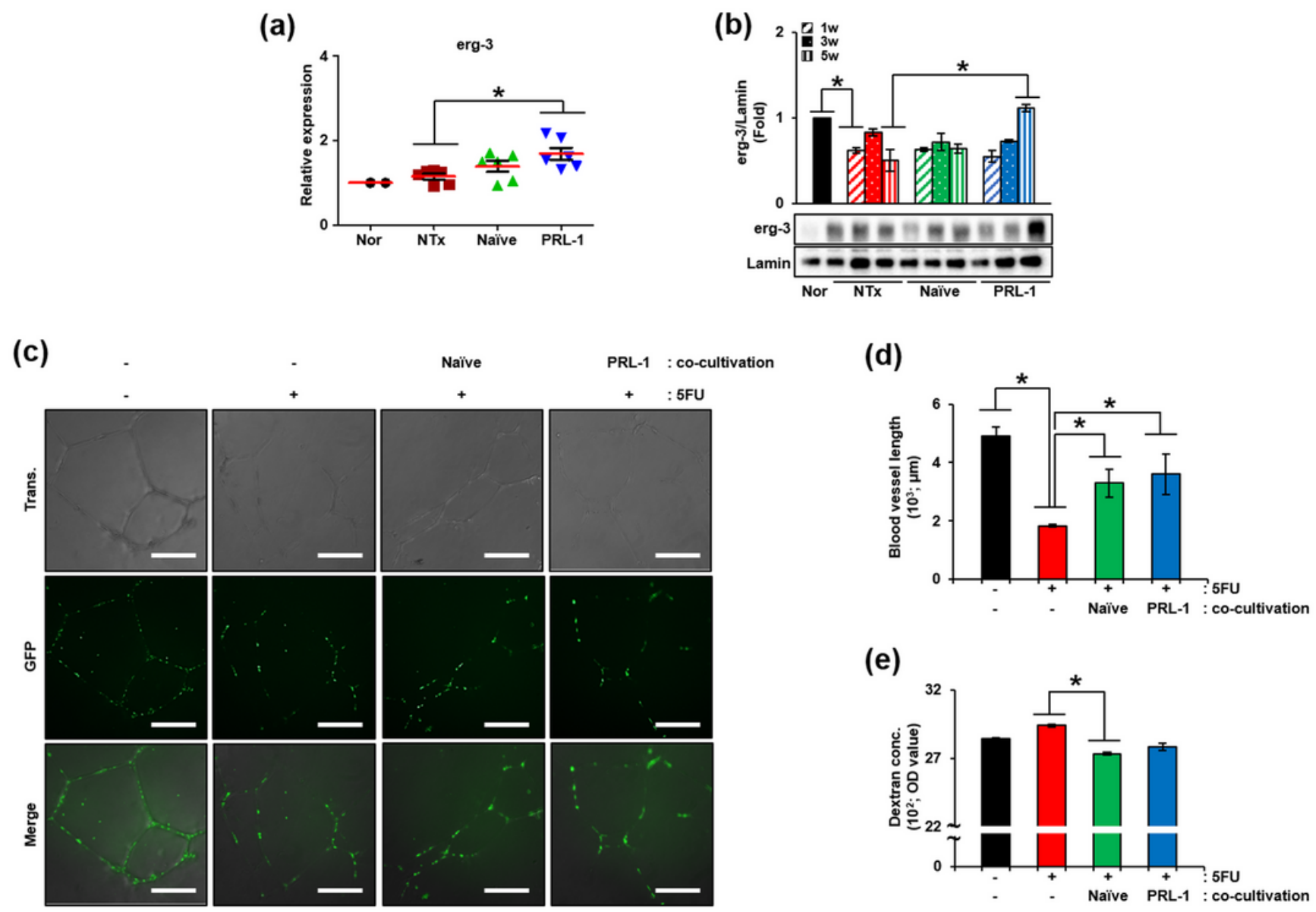

\section{Figure 4}

Effect of transplanted PRL-1-overexpressing cells on vascular permeability in the ovaries of OVX rats. (a) The mRNA expression of erg-3 in ovaries was analyzed by qRT-PCR. (b) The gene expression of erg-3 in the ovary was analyzed by western blots. (c) The vascular formation of HUVECs by PRL- 1 was analyzed by Dil staining. (d) The vascular length of HUVECs was analyzed by ImageJ. (e) The vascular permeability of HUVECs altered by PRL-1 was analyzed by dextran assay. The data are representative of three independent experiments and expressed as the mean \pm S.D. * $p<0.05$, normal vs. NTx, NTx vs. Tx (Naïve and PRL-1), and Naïve vs. PRL-1 at each time point. 
Figure. 5
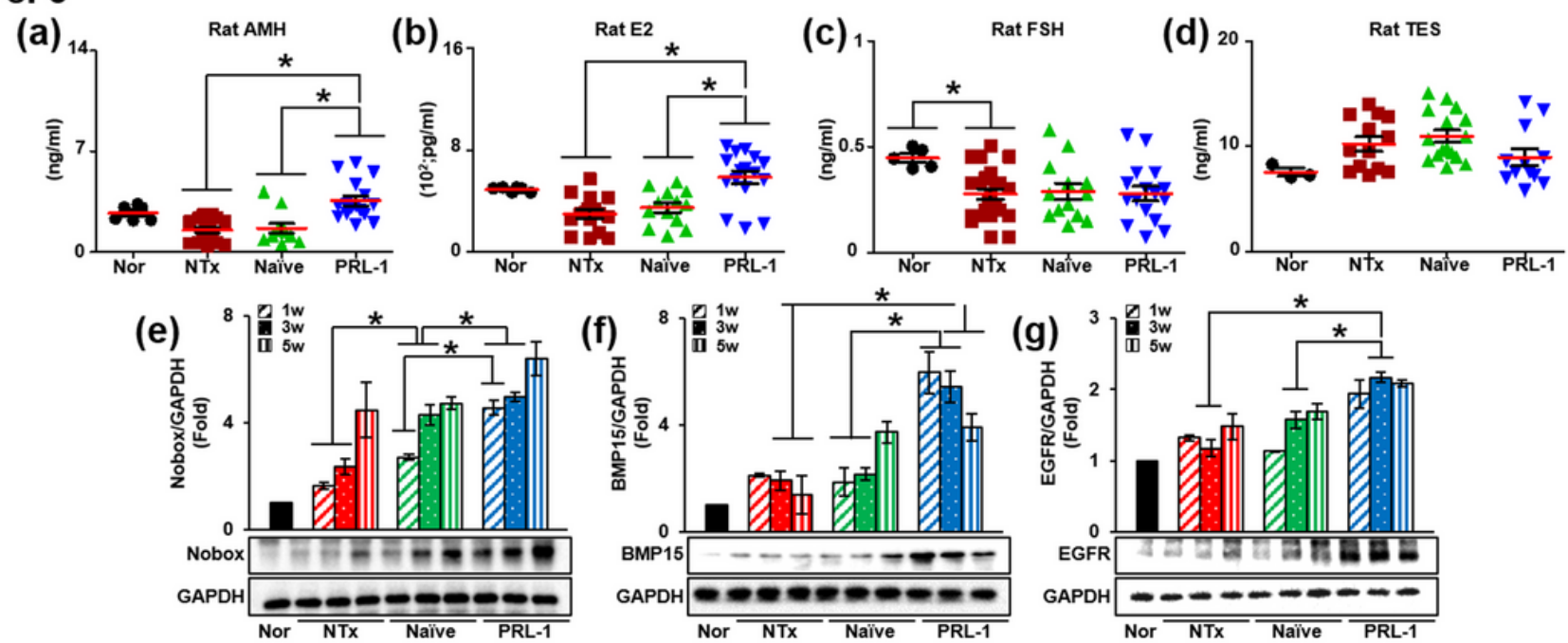

(h)
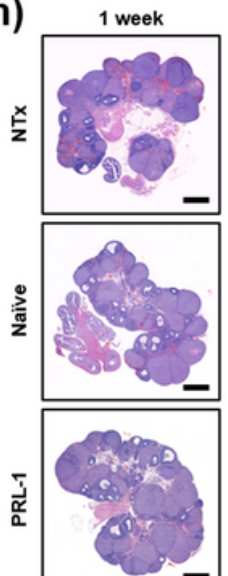

3 weeks

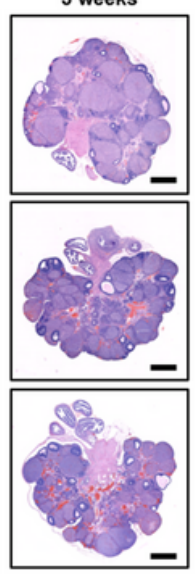

5 weeks

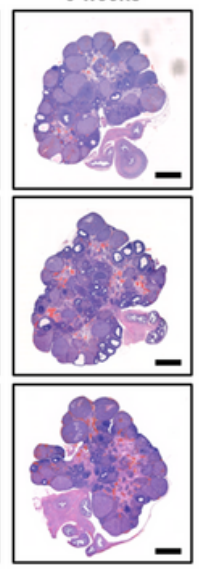

(i)

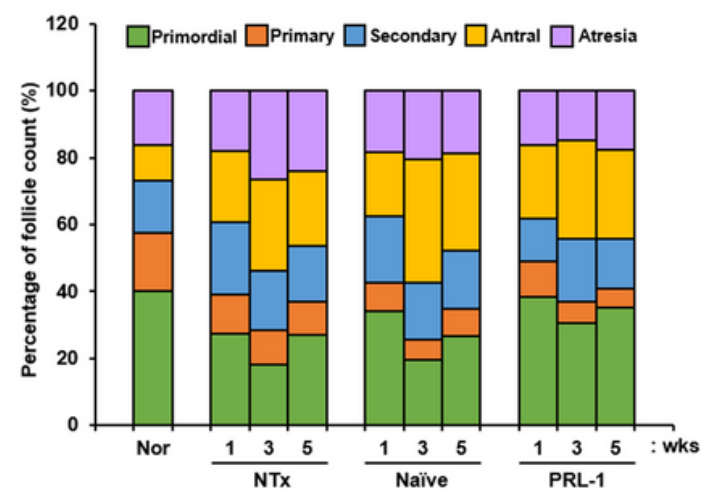

Figure 5

Effect of transplanted PRL-1-overexpressing cells on ovarian function in OVX rats. (a) The levels of AMH, (b) estrogen, (c) FSH and (d) testosterone in individual serum samples were analyzed by ELISAs. (e) The gene expression of Nobox, (f) BMP15 and (g) EGFR in the ovary was analyzed by western blots. (h) Histological analysis of follicular development was analyzed by H\&E staining. Scale bar: $1 \mathrm{~mm}$, magnification: 1.4X. (i) The percentage of follicles according to follicular development was analyzed by the 3D HISTECH program. The data are representative of three independent experiments and expressed as the mean \pm S.D. ${ }^{*} p<0.05$, normal vs. NTx, NTx vs. Tx (Naïve and PRL-1), and Naïve vs. PRL-1 at each time point. 
Figure. 6
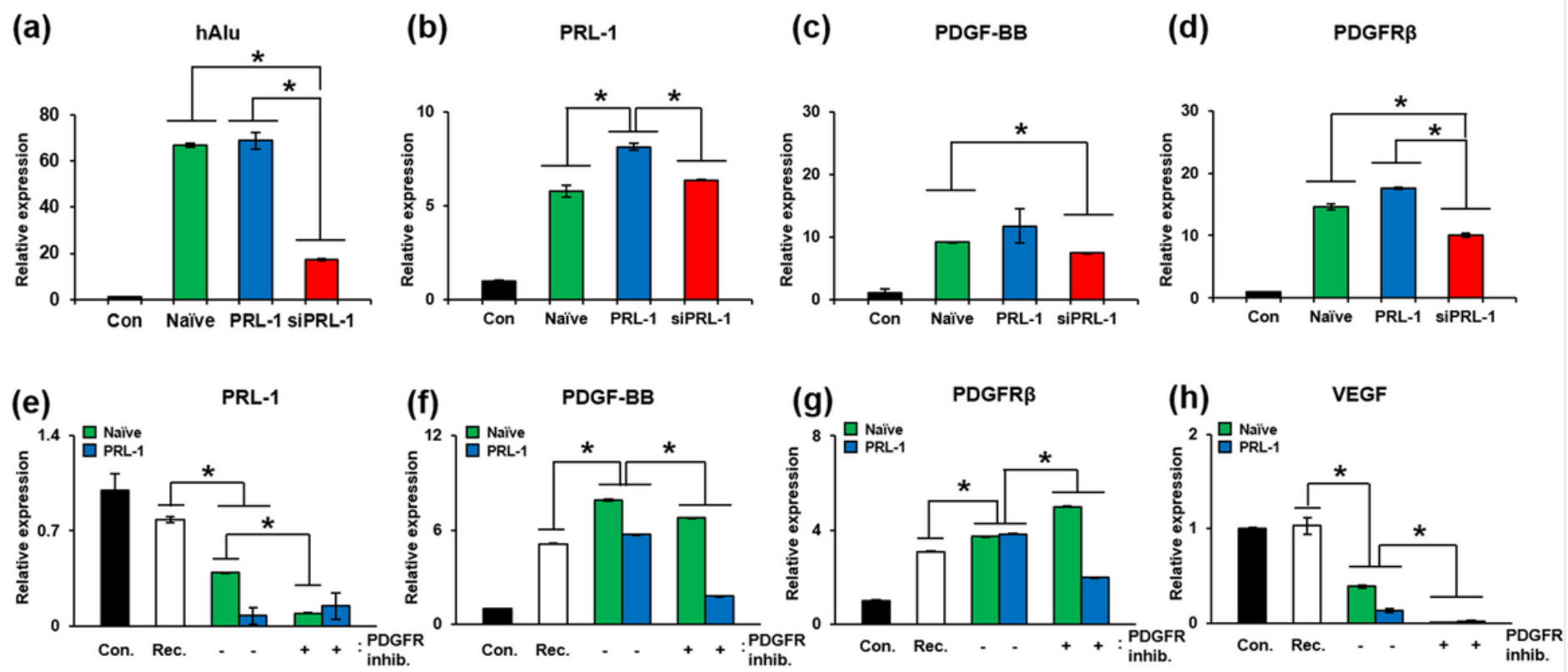

Figure 6

Effect of PRL-1-overexpressign cells on follicular development via the PDGF pathway ex vivo. (a) The mRNA expression of hAlu, (b) human PRL-1, (c) PDGF-BB, (d) PDGFRß, (e) Nobox and (f) Lhx8 in ovaries with siRNA-PRL-1 was analyzed by qRT-PCR. (g) The mRNA expression of PDGF-BB, (h) PDGFRß, (i) VEGF and VEGFR2 in ovaries treated with recombinant PDGF and inhibitor was analyzed by qRT-PCR. The data are representative of three independent experiments and expressed as the mean \pm S.D. * $p<0.05$, normal vs. NTx, NTx vs. Tx (Naïve and PRL-1), Naïve vs. PRL-1 at each time point. 
Figure. 7

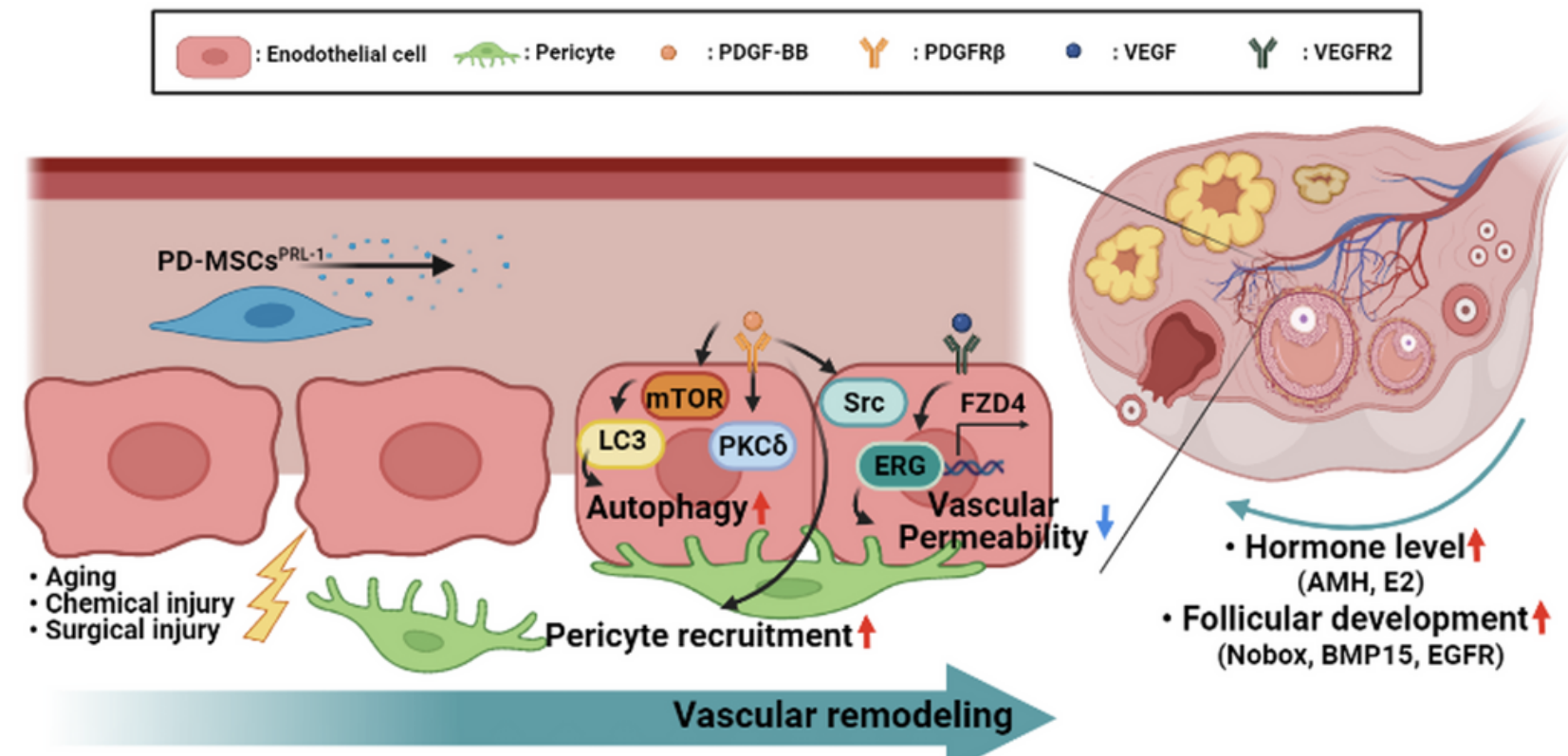

Figure 7

Graphical abstract describes that PRL-1 activates PDGF signaling to improve vascular remodeling in ovarian, thereby improving ovarian function. PD-MSCs, placenta-derived mesenchymal stem cells; PRL-1, phosphatase of regenerating liver-1; PDGF-BB, platelet-derived growth factor-BB; PDGFR $\beta$, platelet-derived growth factor receptor beta; VEGF, vascular endothelial growth factor; VEGFR2, vascular endothelial growth factor receptor 2; mTOR, mammalian target of rapamycin; LC3, microtube-associated protein 1 light chain 3; PKCס, protein kinase C-delta; Src, proto-oncogene tyrosine-protein kinase Src; ERG, ETSrelated gene; FZD4, frizzled class receptor 4; AMH, anti-Mullerian hormone; E2, estrogen; Nobox, newborn ovary homeobox; BMP15, bone morphogenetic protein 15; EGFR, epidermal growth factor receptor

\section{Supplementary Files}

This is a list of supplementary files associated with this preprint. Click to download.

- SCRTSupplementarylnformationParketal.pdf 